\title{
Myocardial blush grade and Thrombolysis in Myocardial Infarction flow grade in ST segment elevation myocardial infarction
}

We have read the article "Predictive value of admission red cell distribution width-platelet ratio for no-reflow phenomenon in acute ST segment elevation myocardial infarction undergoing primary percutaneous coronary intervention" by Celik et al. [1]. The authors have concluded that admission neutrophil-lymphocyte ratio, platecrit, and red cell distribution width-platelet ratio are independent correlates of no-reflow and in-hospital major adverse cardiac events (MACEs) among patients with ST segment elevation myocardial infarction (STEMI) undergoing primary percutaneous coronary intervention (pPCI).

We know that the degree of perfusion in the infarct-related artery is typically described by the Thrombolysis in Myocardial Infarction (TIMI) flow grade. However, the coronary flow velocity pattern in the infarct-related epicardial artery is primarily determined by the microvascular function of the dependent myocardium reflected by the myocardial blush grade (MBG) [2]. The MBG is predictive of outcome in patients with an acute STEMI who are treated with pPCI [3]. It was also previously shown that inflammation seems to be associated with distorted MBG in patients with cardiac syndrome X [4].
Therefore. we are convinced that, if MBG were examined and evaluated with TIMI flow grade, the study could completely reflect the effect of inflammatory parameter on no-reflow and in-hospital MACEs among patients with STEMI undergoing pPCI.

Conflict of interest: None declared

\section{References}

1. Celık T, Balta S, Demır M et al. Predictive value of admission red cell distribution width-platelet ratio for no-reflow phenomenon in acute ST segment elevation myocardial infarction undergoing primary percutaneous coronary intervention. Cardiol J, 2016; 23: 84-92. doi: 10.5603/CJ.a2015.0070.

2. Hoffmann R, Haager P, Lepper W, Franke A, Hanrath P. Relation of coronary flow pattern to myocardial blush grade in patients with first acute myocardial infarction. Heart, 2003; 89: 1147-1151.

3. Gibson CM1, Cannon CP, Murphy SA, Marble SJ, Barron HV, Braunwald E. Relationship of the TIMI myocardial perfusion grades, flow grades, frame count, and percutaneous coronary intervention to long-term outcomes after thrombolytic administration in acute myocardial infarction. Circulation, 2002; 105: 1909-1913.

4. Okyay K, Yilmaz M, Yildirir A, Eroglu S, Sade E, Sahinarslan A, Relationship between neutrophil-to-lymphocyte ratio and impaired myocardial perfusion in cardiac syndrome X. Eur Rev Med Pharmacol Sci, 2015; 19: 1881-1887.

Sinan İscen

Diyarbakir Military Hospital, Diyarbakir, Turkey e-mail:dr.iscen@hotmail.com 